\title{
11 ADOLESCENTE COM COMPORTAMENTO AUTOLESIVO SEM INTENÇÃO SUICIDA NO INTERNAMENTO DO SERVIÇO DE URGÊNCIA DE UM HOSPITAL PEDIÁTRICO DA REGIÃO CENTRO
}

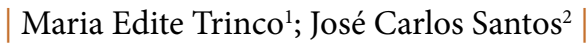

\section{RESUMO}

CONTEXTO: A adolescência é reconhecidamente um período de transição entre a infância e a idade adulta, onde ocorrem múltiplas transformações físicas, psíquicas, sociais, familiares, que exigem do adolescente uma permanente adaptação a esta fase do desenvolvimento.

OBJETIVO(S): caracterizar os adolescentes com comportamento autolesivo que recorreram no serviço de urgência de um hospital pediátrico; categorizar os comportamentos autolesivos; identificar o motivo que levou ao comportamento autolesivo.

MÉTODOLOGIA: estudo qualitativo, descritivo, tendo como base de pesquisa o estudo de casos múltiplos.

RESULTADOS: Da análise dos dados podemos inferir que as raparigas com uma média de idade de 15,5 anos são quem mais tem comportamentos autolesivos. As intoxicações medicamentosas são as que têm maior relevância, seguido das automutilações. Sendo que 55\% dos adolescentes referem que o motivo para o ato é o sofrimento psíquico em que se encontram, 39,5\% referem disfunção familiar, a escola e as relações entre pares também aparecem como motivo para o ato.

CONCLUSÃO: O adolescente que tem um comportamento autolesivo sem intenção suicida está em sofrimento psíquico, e utiliza este comportamento para aliviar esse mesmo sofrimento. As raparigas são quem mais adota este tipo de comportamento, com a ingestão medicamentosa voluntária a prevalecer em relação aos restantes métodos.

\section{PALAVRAS-CHAVE: adolescente, comportamento autodestrutivo; emergência}

\section{RESUMEN}

"El adolescente con comportamiento autolesivo no suicida en el ingreso en el servicio de urgencias de un hospital pediátrico de la región centro"

CONTEXTO: La adolescencia se considera un periodo de transición entre la infancia y la edad adulta, en el que se producen múltiples transformaciones físicas, psíquicas, sociales y familiares, que exigen al adolescente una permanente adaptación a esta fase del desarrollo.

OBJETIVO(S): caracterizar a los adolescentes con comportamiento autolesivo que ingresaron en el servicio de urgencias de un hospital pediátrico; clasificar los comportamientos autolesivos; identificar el motivo que provocó el comportamiento autolesivo.

METODOLOGÍA: estudio cualitativo, descriptivo, que tiene como base de investigación el estudio de casos múltiples.

RESULTADOS: del análisis de los datos podemos deducir que las niñas con una media de edad de 15,5 años son las que muestran más comportamientos autolesivos. Las intoxicaciones medicamentosas presentan una mayor relevancia, seguidas de las automutilaciones. El $55 \%$ de los adolescentes señala como motivo para tal comportamiento el sufrimiento psíquico que soportan, mientras que el 39,5 \% señala la disfunción familiar; el colegio y las relaciones con sus compañeros también aparecen entre los motivos.

CONCLUSIONES: el adolescente con comportamiento autolesivo no suicida padece sufrimiento psíquico y utiliza este comportamiento para aliviar dicho sufrimiento. Las niñas adoptan con mayor frecuencia este tipo de comportamiento y la ingesta medicamentosa voluntaria prevalece sobre los demás métodos.

DESCRIPTORES: adolescente; comportamiento autodestructivo; emergencia

\begin{abstract}
"Teenagers with self-injurious behaviour and no suicidal intention in the ER of a paediatric hospital in the centre region of the country"

BACKGROUND: Youth is recognised as a transition period between child and adulthood, when several physical, psychic, social, family changes take place, which require of the teenager adjustment to this stage of his development.

AIM: Characterising teenagers with self-injurious behaviour who were assisted in emergency in a paediatric hospital; classifying self-injurious behaviour; identifying the motives for self-injurious behavior.

METHODS: qualitative, descriptive study, with research based on multiple case studies.

RESULTS: One infers that girls with an average age of 15.5 years show self-injurious behaviour the most. Drug poisoning is at the top of the list, followed by selfmutilation. As for the motives, $55 \%$ of teenagers mention the psychological distress they find themselves in, $39.5 \%$ allude to family dysfunction, while school and peer relations are also given as reasons. CONCLUSIONS: The teenager with self-injurious behaviour and no suicidal intention is in psychological distress, and resorts to such behaviour to alleviate the suffering. This is more common among girls, with voluntary drug administration prevailing over the other methods.
\end{abstract}

\section{KEYWORDS: teenager; self-destructive behavior; emergency}

Submetido em 29-01-2017

Aceite em 20-05-2017

1 Mestre em psicossociologia da saúde, enfermeira especialista em saúde infantil e pediátrica, centro hospitalar e universitário de Coimbra-hospital pediátrico, serviço de pedopsiquiatria. Urb. Ferreira Jorge, 3040-016 Coimbra, Portugal, edite.trinco@hotmail.com

2 Doutorado em Saúde Mental, Professor coordenador na Escola Superior de Enfermagem de Coimbra. 3046-851 Coimbra, Portugal, jcsantos@esenfc.pt

Citacão: Trinco, M. E., \& Santos, J.C. (2017). O adolescente com comportamento autolesivo sem intenção suicida no internamento do serviço de urgência de um hospital pediátrico da região centro. Revista Portuguesa de Enfermagem de Saúde Mental (Spe. 5), 63-68. 


\section{INTRODUÇÃO}

O início e o términus da adolescência está longe de obter consenso. Segundo a Organização Mundial de Saúde, a adolescência abrange o período do ciclo vital que vai desde os 10 aos 19 anos. (WHO, 2011). É um período em que o adolescente revela um modo de vida muito peculiar transitando num longo trajeto entre o final da infância até ao início da idade adulta, onde as alterações físicas e psicossociais estão estritamente sujeitas e influenciadas pela interação do adolescente com o meio envolvente e seus contextos (Shaffer \& Kipp, 2007; Sampaio, 2006).

A adolescência é reconhecidamente um período de transição entre a infância e a idade adulta, onde ocorrem múltiplas transformações físicas, psíquicas, sociais, familiares, que exigem do adolescente uma permanente adaptação a esta fase do desenvolvimento, prevalecendo a organização e reorganização da sua própria identidade (Brito, 2011). Este processo decorre habitualmente de forma segura e saudável no desenvolvimento da sua complexidade (Salgado \& Neves, 2011; WHO, 2005). Contudo, durante esta transição, o adolescente depara-se com desafios e conflitos, e, o modo de resposta interna e externa aos mesmos, vai influenciar substancialmente o sucesso ou o fracasso desta evolução do ciclo da vida (Santos \& Neves, 2014). Podendo assim inferir-se que a adolescência representa um período de metamorfose individual, onde o adolescente constrói o seu processo de vida com o intuito de atingir a sua autonomia e responsabilidade, perfazendo o caminho de resolução das múltiplas tarefas desenvolvimentais, procurando a consolidação da sua própria identidade. (Santos et al,2014).

É nesta transição, que por vezes o adolescente sente dificuldade de ajuste saudável e uma necessidade de procura incessante da construção da identidade que poderão ter a sua origem nos mais variados fatores psicológicos, biológicos, familiares e sociais, subsistindo um modelo de causalidade multifatorial complexa, que necessita de uma compreensão adequada de forma a ajudar o adolescente na resolução dos seus conflitos, mitigando assim a probabilidade da adoção de comportamentos autolesivos (Whitlock, 2010; Sociedade Portuguesa de Suicidologia, 2014).

Ao longo do tempo a literatura tem sido pouco consensual e consistente na definição dos comportamentos autolesivos, assim como na diferenciação destes comportamentos com características diferentes.
Esta definição tem comportado termos como: parassuicido, automutilação, cutting, violência autodirigida, selfmutilation, deliberate self harm, self injury, self inflicted violence (Rodham \& Hawton, 2009; Klonsky et al, 2014).

Apesar da dificuldade de consenso na definição é transversal aos vários autores de que se trata de: um ato não fatal exercido pelo adolescente, sem intervenção de outros, em que deliberadamente ingere uma substância em excesso de dosagem terapêutica, tem um comportamento com intenção de causar lesão ao próprio corpo (cortes, queimadura) ingere uma substancia ilícita ou outra não ingerível (DGS, 2013).

Da literatura por nós revisitada, verificamos que os comportamentos autolesivos apresentam uma maior prevalência na adolescência (Laye-Gindhu \& Schonert-Reichl,2005; Sampaio, 2006; Matos et. al., 2012), com tendência para a remissão natural na idade adulta. É de salientar que os métodos mais utilizados nestes comportamentos são as intoxicações e sobredosagens medicamentosas assim como as automutilações (Santos, 2000; Kapur et al, 2013), sendo mais frequentes no género feminino (Guerreiro et al 2014; Zetterqvist, 2015), contrastando com o comportamento suicida, que é mais relevante no género masculino (Santos, 2000; Shaffer et al, 2001; Sampaio, 2009).

Os comportamentos autolesivos em adolescentes são considerados um problema de saúde pública necessitados de entendimento e acompanhamento na sua complexa interação biopsicossocial, relacional e familiar devido ao seu manifesto crescimento sobretudo entre adolescentes comprometendo um desenvolvimento saudável (Trinco e Santos, 2015; Guerreiro et al, 2014; Muehlenkamp et al, 2012; Nock \& Favazza, 2009). A nossa prática é concordante com os autores, assim sendo e perante esta problemática compete ao enfermeiro compreender como é que os adolescentes vivem estas situações. E é nesta senda de vulnerabilidade e mudança com as quais o adolescente ainda não sabe viver harmoniosamente que poderá surgir a adoção de comportamentos de risco (Vrouva et al., 2010) entre eles os comportamentos autolesivos, que podem servir de modelo de regulação emocional como forma de expressar e externalizar emoções intensas (Nock, Prinstein \& Sterba, 2009), ou por outro lado, são utilizados como um meio de comunicação, quando existe um défice na mesma ou simplesmente ela não existe, dependendo das condições e da capacidade de resposta às necessidades internas, readaptações externas e dos meios de ajuda do contexto familiar, afetivo, académico e sociocultural (Trinco \& Santos, 2015). 
De acordo com a DGS (2013), os comportamentos autolesivos são frequentes em adolescentes, sobretudo nas raparigas. Mas os dados epidemiológicos são parcos, deixando assim uma lacuna na verdadeira realidade deste flagelo. Os estudos de Gmitrowlcz, et al. (2014) e de Morey, et al. (2008), dizem-nos que apenas $14 \%$ dos adolescentes procurou os cuidados de saúde após um comportamento autolesivo. Já para Guerreiro, et al (2014), só 13\% recorreram ao hospital, essencialmente nos casos de intoxicações. Assim sendo, crê-se que seja um problema de grande relevância, carente de investigação (Cordovil. et al, 2009).

Perante tais considerações delineámos como objetivos: caracterizar os adolescentes com comportamento autolesivo que recorreram no serviço de urgência; categorizar os comportamentos autolesivos; e identificar o motivo que levou ao comportamento autolesivo, de forma a identificamos os adolescentes em risco de comportamentos da esfera suicidária, possibilitandonos um cuidado holístico.

\section{METODOLOGIA}

Para a consecução dos objetivos e atendendo à natureza do fenómeno que se pretende investigar, a investigação direciona-se para a metodologia qualitativa de orientação para um estudo de casos múltiplos, em cuja pesquisa será desenvolvida a análise de entrevista aos pais que acompanharam o filho durante este internamento, processo clinico do adolescente e notas de campo do investigador.

As entrevistas tiveram como base um guião composto por uma caracterização sociodemográfica da família e uma pergunta aberta para perceber o motivo do adolescente para o comportamento.

O estudo de caso como método de pesquisa permite ao investigador utilizar múltiplas fontes de evidência, e quando a investigação coloca questões do tipo "como" e porquê", de forma que os dados convirjam para uma melhor análise em profundidade do objeto de investigação de forma holística preservando as características significativas dos acontecimentos da vida real (Yin,2001).

\section{População e amostra}

O estudo decorreu no serviço de urgência de um hospital pediátrico da região centro, de Janeiro a Novembro de 2015, com um total de admissões de 55507 crianças e adolescentes, sendo a população adolescente, com idade compreendida entre os 13 e os 17 anos e 364 dias, de 5 163, dos quais 413 são adolescentes com sofrimento mental.
A amostra é constituída por 38 adolescentes, provenientes dos distritos de Coimbra, Aveiro, Leiria e Guarda, que tiveram uma intoxicação medicamentosa voluntária e/ou uma automutilação sem intenção suicida que ficaram internados no referido serviço.

\section{Procedimento de colheita de dados}

Os instrumentos de colheita de dados foram a entrevista semiestruturada aos pais, após cumprimento de todas as formalidades ético-deontológicas, o processo clinico do adolescente e as notas de campo.

Para perceção mais profícua de todos os dados, foi utilizada a técnica de análise de conteúdo, com apoio do programa informático NVIVO8.

\section{RESULTADOS}

Parece-nos pertinente caracterizar o adolescente que teve o comportamento autolesivo, para uma melhor e mais abrangente compreensão do contexto.

Quadro 1 - Caracterização do adolescente segundo o género

\begin{tabular}{|l|l|l|}
\hline Adolescente & $\mathbf{n}$ & \% \\
\hline Raparigas & 30 & 78,94 \\
\hline Rapazes & 8 & 21,06 \\
\hline Total & 38 & 100,00 \\
\hline
\end{tabular}

Relativamente ao género, são as raparigas quem mais tem comportamentos autolesivos, o que está de acordo com os estudos nacionais e internacionais.

Quadro 2 - Caracterização do adolescente segundo a idade

\begin{tabular}{|l|l|l|}
\hline Idade do adolescente & $\mathbf{n}$ & \% \\
\hline 13 anos & 6 & 15,79 \\
\hline 14 anos & 6 & 15,79 \\
\hline 15 anos & 9 & 23,68 \\
\hline 16 anos & 9 & 23,68 \\
\hline 17 anos & 8 & 21,06 \\
\hline Total & 38 & 100,00 \\
\hline
\end{tabular}

No que respeita à idade do adolescente, a média situase nos 15,5 anos. Os estudos de Ferrey et al (2015), também obtiveram dados idênticos.

Quadro 3 - Caracterização do motivo de internamento

\begin{tabular}{|l|l|l|}
\hline Motivo do internamento & $\mathbf{n}$ & \% \\
\hline Intoxicação & 32 & 84,21 \\
\hline Automutilação & 6 & 15,79 \\
\hline Intoxicação + automutilação & 20 & 52,63 \\
\hline
\end{tabular}


Quanto ao motivo do internamento, as intoxicações medicamentosas têm maior predominância com $84,21 \%$ do total dos internamentos. Contudo não podemos deixar de referir que $52,63 \%$ tiveram dois ou mais comportamentos associados no mesmo momento, com um tempo médio de internamento de 5 dias. Similarmente os estudos de Santos (2000), Cordovil, et al (2009) e Ferrey et al (2015), chegaram a resultados semelhantes.

Quadro 4 - Caracterização da amostra segundo a proveniência

\begin{tabular}{|l|l|l|}
\hline Proveniência & N & \% \\
\hline Coimbra & 28 & 73,68 \\
\hline Aveiro & 4 & 10,52 \\
\hline Leiria & 3 & 7,90 \\
\hline Guarda & 3 & 7,90 \\
\hline Total & 38 & 100,00 \\
\hline
\end{tabular}

Os adolescentes são provenientes da região centro do país (Coimbra, Aveiro, Leiria e Guarda), sendo Coimbra o distrito com maior relevância $(73,68 \%)$, muito pelo facto de ser este o hospital de referência da área de residência.

A análise dos dados visou obter contributos para uma melhor compreensão sobre o que leva um adolescente a ter um comportamento autolesivo sem intenção suicida. Da pesquisa sobressaiu o sofrimento psíquico como categoria principal, que se subdividiu em três subcategorias: disfunção familiar, escola e relação entre pares.

\section{DISCUSSÃO}

\section{Sofrimento psíquico}

O comportamento autolesivo é um fenómeno multideterminado, ou seja, existem múltiplos fatores que concorrem para a concretização do mesmo, contudo e é transversal a todos os estudos nacionais e internacionais que na sua génese está o sofrimento psíquico do adolescente que vê neste comportamento a fuga à sua dor (Vieira et al, 2016).

\section{Disfunção familiar}

Nesta subcategoria sobressaem os conflitos familiares como fator desencadeante para o ato, não havendo diferenciação em relação ao sexo. Os adolescentes têm sobretudo discussões com os pais e ciúmes dos irmãos, também os problemas financeiros foram referidos como motivo para agravar os conflitos familiares.
Os resultados obtidos no estudo reforçam os dados de Gmitrowlcz, et al. (2014) e Lundh et al, (2011), tendo a disfunção familiar um maior relevo nos comportamentos autolesivos sem intenção suicida.

\section{Escola}

A escola assume uma área de transição na vida do individuo, tornando-se fundamental sobretudo na adolescência, sendo o elo entre dois mundos, o grupo familiar e o grupo social (Marcelli \& Braconnier, 2005). Estes resultados são concordantes com o estudo de Brito \& Oliveira (2013), que nos dizem que existe uma correlação positiva entre: bullying, angústia, baixa autoestima e a perda de confiança do adolescente, podendo ainda verificar-se o abandono escolar e/ou comportamentos autolesivos e em casos extremos o suicídio.

\section{Relação entre pares}

A escolha do grupo e a sua aproximação são cruciais na adolescência para, simultaneamente, entrepor algum distanciamento entre a família e a aprendizagem de socialização tão necessária ao seu futuro, mas, simultaneamente é um fator de risco, quando esta escolha não é bem conseguida (Braconnier \& Marcelli,2000). Foi verificada uma conexão expressiva entre a presença de comportamento autolesivo e a existência de conflito entre pares, sobretudo na rutura do namoro e dificuldade na socialização.

\section{CONCLUSÃO}

O adolescente que tem um comportamento autolesivo sem intenção suicida está em sofrimento psíquico, mas a abordagem não deve ser centrada somente no comportamento ou no sintoma, tem que ser abrangente, porque estes comportamentos são muitas vezes a ponta do iceberg da desesperança destes adolescentes.

Dos adolescentes internados, foram as raparigas com uma idade média de 15,5 anos, quem mais adotou este tipo de comportamento, com a ingestão medicamentosa voluntária a prevalecer em relação aos restantes métodos e os conflitos familiares na sua origem. A maioria referiu que o comportamento serviu para atenuar a dor sentida naquele momento.

O internamento destes adolescentes é um momento de excelência para a compreensão do comportamento autolesivo na adolescência, tornando-se essencial, para definir estratégias que proporcionem ao adolescente um crescer saudável, onde a crise individual, social e familiar estão intricadas e poderão ser promotoras da sua autorregulação. 


\section{IMPLICAÇÕES PARA A PRÁTICA CLÍNICA}

Assim, salienta-se a relevância do estudo e da sua continuidade para delinear intervenções pertinentes para um melhor cuidar destes adolescentes e prevenção de recidivas.

\section{REFERÊNCIAS BIBLIOGRÁFICAS}

Braconnier, A., \& Marcelli, D. (2000). As mil faces da adolescência. Lisboa. Climepsi Editores.

Brito, C., \& Oliveira, M. (2013). Bullying and self-esteem in adolescents from public schools. Jornal de Pediatria. 89 (6): 601-607. doi: 10.1016/j.jped.2013.04.001

Brito, I. (2011). Ansiedade e depressão na adolescência. Dossier: adolescência. Revista Portuguesa de Clinica Geral. 27(2): 208-214.

Cordovil, C., Crujo, M., \& Guerreiro, D. (2009). Tentativas de Suicídio em Adolescentes Internados na Unidade de Internamento de Pedopsiquiatria do Centro Hospitalar de Lisboa Central. Rev. de Saúde Mental. On line in: repositorio.chlc.min-saude.pt/...17/.../ Rev\%20Saude\%20Mental\%202009_XI_15.pdf. em 2016.08.23.

Ferrey, A., Hughes, N., Simkin, S., Locock, L., Stewart, A., Kapur, N., \& Hawton, K. (2015). The impact of self-harm by young people on parents and families: a qualitative study. BMJ open, 6 (1), doi: http://dx.doi. org/10.1136/bmjopen-2015-009631

Gmitrowicz, A., Kostulski, A., Kropiwnicki, P., \& Zalewska-Janowska (2014) A. Cutaneous deliberate selfharm in Polish school teenagers - an inter-disciplinary challenge. Acta Dermato-Venereologica.; 94(4): 448453. doi: 10.2340/00015555-1690.

Guerreiro, D.F., Sampaio, D., \& Figueira, M.L. (2014). Relatório de investigação "Comportamentos autolesivos em adolescentes: características epidemiológicas e análise de fatores psicopatológicos, temperamento afectivo e estratégias de coping". Clinica Universitária de Psiquiatria e Psicologia. Universidade de Lisboa. On line in: http://www.spsuicidologia.pt/generalidades/ biblioteca/143. relatorio da investigação comportamentos autolesivos em adolescentes. em 2016.09.14.
Laye-Gindhu, A., \& Schonert-Reichl, K.A. (2005). Nonsuicidal self-harm among community adolescents: understanding the "whats" and "whys" of self-harm. Journal of Youth Adolescence. 34: 447. doi:10.1007/ s10964-005-7262-Z

Lundh, L. G., WÅngby-Lundh, M., \& Bjärehed, J. (2011). Deliberate self-harm and psychological problems in young adolescents: Evidence of a bidirectional relationship in girls. Scandinavian journal of psychology, 52(5), 476-483. doi:10.1111/j.14679450.2011.00894.x

Klonsky, E. D., Victor, S. E., \& Saffer, B. Y. (2014). Nonsuicidal Self-Injury: What We Know, and What We Need to Know. Canadian Journal of Psychiatry. Revue Canadienne de Psychiatrie, 59(11), 565-568.

Matos, M.G., Simões, C., Tomé, G., Camacho, I., Ferreira, M., \& Ramiro, L. (2012). A saúde dos adolescentes portugueses: relatório do estudo HBSC. Lisboa: Centro de Malária e outras Doenças Tropicais/IHMT/ UNL.

Marcelli, D., \& Braconnier, A. (2005). Adolescência e psicopatologia. Lisboa: Climepsi Editores.

Monteiro, P., \& Confraria, L. (2014). Adolescência. In P. Monteiro (Coord.), Psicologia e Psiquiatria da Infância e Adolescência (pp. 339-358). Lisboa: Lidel Edições Técnicas, Lda.

Morey, C., Corcoran, P., Arensman, E., \& Perry, I.J. (2008). The prevalence of self-reported deliberate self harm in Irish adolescents. BMC Public Health; 8: 79. doi: 10.1186/1471-2458-8-79.

Muehlenkamp, J., Claes, L., Havertape, L., \& Plener, P. L. (2012). International prevalence of adolescent nonsuicidal self-injury and deliberate self-harm. Child and Adolescent Psychiatry and Mental Health, 30(6), 10. doi: 10.1186/1753-2000-6-10.

Nock, M., \& Favazza, A. (2009). Non-suicidal self-injury: Definition and classification. In M. K. Nock (Ed.), Understanding non-suicidal self-injury: Origins, assessment, and treatment (pp. 9-18). Washington, DC: American Psychological Association. 
Portugal, Direcção Geral da Saúde. (2013). Plano Nacional de Prevenção do Suicído 2013/2017. On line in: http://www.portaldasaude.pt/NR/rdonlyres/ BCA196AB-74F4-472B-B21E6386D4C7A9CB/0/ i018789.pdf.

Rodham, K., \& Hawton, K. (2009). Epidemiology and phenomenology of nonsuicidal self-injury. In: Nock MK, editor. Understanding Nonsuicidal Self-Injury: Origin, Assessment, and Treatment. Washington, DC: American Psychological Association. pp. 37-62.

Salgado, M., \& Neves, E. (2011). Um olhar sobre o para-suicídio na adolescência. Revista Portuguesa de Pedopsiquiatria, 30, 53-58.

Sampaio, D. (2009), Ninguém morre sozinho. 15ª Edição. Lisboa: Caminho.

Sampaio D. (2006). Lavrar o mar. Lisboa: Editorial Caminho.

Santos, N., \& Neves, E.; (2014). Adolescência e comportamentos suicidários. Suicídio e comportamentos autolesivos. Dos conceitos à prática clínica. Lisboa: Lidel.

Santos, J.C. P., Erse, M.P.Q.A., Marques, L.A.F.A., Façanha, J. D. N., Marques, L. A. F. A., \& Simões, R. M. P. (2014). Contigo: promoção da saúde mental e prevenção do comportamento suicidário na comunidade educativa. Série Monográfica Educação e Investigação em Saúde.

Santos (2000) O para-suicídio no Concelho de Coimbra: contributos para o seu estudo. Revista Sinais Vitais. 28,15-19.
Shaffer, D., \& Kipp, K. (2007). Developmental Psychology Childhood and Adolescence. Belmont: Wadworth. Sociedade Portuguesa de Suicidologia. (2014). Suicídio e Comportamentos Autolesivos. Dos conceitos à prática. Lidel-edições técnicas. Lisboa.

Trinco. E., \& Santos. J.C. (2015) O adolescente com alteração do comportamento no serviço de urgência: estudo de um quadriénio. Revista Investigação em Enfermagem, 13 Série 2.

Vieira, M., Pires, M., \& Pires, O. (2016). Self-mutilation: pain intensity, triggering and rewarding factors. Revista Dor, 17(4), 257-260.

Vrouva, I., Fonagy, P., Fearon, P. R. M., \& Roussow, T. (2010). The risk-taking and self-harm inventory for adolescents: Development and psychometric evaluation. Psychological Assessment, 22(4), 852-865. doi: 10.1037/a0020583

WHO (World Heatlh Organization) (2011). Adolescent health. Online in www.who.int/topics/adolescent_health/en em 2016.03.22.

WHO, (World Heatlh Organization) (2005). Promoting Mental Health concepts emerging evidence practice. Online in: http://www.who.int/mental_health/ evidence/MH_Promotion_Book.pdf. em 2016.07.25.

Yin, R. (2001). Case Study Research: Design and Methods ( $5^{\mathrm{a}}$ Ed) Thousand Oaks, CA: SAGE Publications.

Zetterqvist, M. (2015). Child and Adolescent Psychiatry and Mental Health. 9:31 doi: 10.1186/s13034-0150062-7. 